\title{
Some Results for the Existence of Periodic Solutions to $p$-Laplacian Equation on Time Scales
}

\author{
Dianwu Yang, Fengjuan Cao, Zhenlai Han* \\ School of Mathematical Sciences, \\ University of Jinan, \\ Jinan, Shandong, 250022, P R China
}

\begin{abstract}
In this paper, we investigate a class of Lié nard type p-Laplacian equation on times scales by generalized Mawhin's continuation theorems, under suitable conditions, we ensure that at least one periodic solution to this kind of p-Laplacian equation on time scales exist.
\end{abstract}

Keywords-generalized mawhin's continuation theorems; pLaplacian; periodic solution; time scales.

\section{INTRODUCTION}

Li é nard equations can be derived from many fields, such as mechanics, engineering technique fields, physics, and so on, and is important in describing fluid mechanical and nonlinear elastic mechanical phenomena.

Many authors have contributed to the theory of the equations with respect to existence of periodic solutions (see e.g. [1-6] and the reference therein), during the past several years.

The important and useful tools to study this class of differential equations are Mawhin's continuation theorem, generalized polar coordinates, Leary-Schauder degree theory and many fixed point theory.

Mawhin's continuation theorems has been extensivly used for getting the existence of periodic solutions to this class equation.

For example, using Mawhin's continuation theorem, Cheung and Ren considered the existence of T-periodic solutions to a Li é nard type $p$-Laplacian equation with a deviating argument in [7],

$$
\left(\varphi_{p}\left(x^{\prime}(t)\right)\right)^{\prime}+F(x(t)) x^{\prime}(t)+G(x(t-\tau(t)))=E(t),
$$

and some results for the existence of periodic solutions were got. Lu investigated the existence of periodic solutions for a $p$-Laplacian Li é nard differential equation with a deviating argument by using Mawhin's continuation theorem in [8].

Du and Zhao [9] introduce us the existence of periodic solution to a $p$-Laplacian Li é nard equation by means of generalized Mawhin's continuation theorem. Although the results of this class of differential equation are plentiful, the argument of periodic solutions on time scales hasn't got much attention, see $[4,5,10-14]$.
In [11], Li and Zhang considered the periodic solutions for a periodic mutualism model on a time scale $T$ by employing Mawhin's continuation theorem, and obtained three sufficient criteria.

In this paper, we will systematically investigate the existence of periodic solutions of the Lié nard $p$-Laplacian equation

$$
\left(\varphi_{p}\left(x^{\Delta}(t)\right)\right)^{\Delta}+f(x(t)) x^{\Delta}(t)+g(x(t-\tau(t)))=e(t)
$$

on a time scales $T$. Our technique is motivated by that used in [14], and we applying it to investigate the existence of periodic solutions for (1.1).

The setup of this paper is as following. In the coming section, we present some lemmas and definitions on time scales. In Section 3, we systematically explore the existence of periodic solutions of the Li é nard type $p$-Laplacian equation on time scales.

\section{PRELIMINARY}

In this section, we briefly give some basic definitions, lemmas on time scales which are used in the follows. Let $T$ be a time scale (a nonempty closed subset of $R$ ). The forward and backward jump operators $\sigma, \rho: T \rightarrow T$ and the graininess $\mu: T \rightarrow R^{+}$.

Definition 2.1. ([15]) Let $X$ and $Z$ be two Banach spaces with norms $\|\cdot\|_{X},\|\cdot\|_{Z}$, respectively. A continuous operator $\quad M: X \cap \operatorname{dom} M \rightarrow Z$

is said to be quasi-linear if

(i) $\operatorname{Im} M:=M(X \cap \operatorname{dom} M)$ is a closed subset of Z;

(ii) $\operatorname{Ker} M:=\{x \in X \cap \operatorname{dom} M: M x=0\} \quad$ is linearly homeomorphic to $R^{n}, n<\infty$.

Definition 2.2. ([15]) Let $\Omega \subset X$ be an open and bounded set with the origin $\theta \in \Omega . \quad N_{\lambda}: \bar{\Omega} \rightarrow Z$, $\lambda \in[0,1]$ is said to be $M-$ compact in $\bar{\Omega}$ if there exists subset $Z_{1}$ of $Z$ satisfying $\operatorname{dim} Z_{1}=\operatorname{dimKerM}$ and an 
operator $R: \bar{\Omega} \times[0,1] \rightarrow X_{2}$ being continuous and compact such that for $\lambda \in[0,1]$,

(a) $(I-Q) N_{\lambda}(\bar{\Omega}) \subset I m M \subset(I-Q) Z$,

(b) $Q N_{\lambda} X=0, \lambda \in(0,1) \Leftrightarrow Q N x=0$,

(c) $R(\cdot, 0) \equiv 0$ and $\left.R(\cdot, \lambda)\right|_{\Sigma_{\lambda}}=\left.(I-P)\right|_{\Sigma_{\lambda}}$,

(d) $M(P+R(\cdot, \lambda)]=(I-Q) N_{\lambda}, \lambda \in[0,1]$,

where $X_{2}$ is the complement space of $\operatorname{KerM}$ in $X$, i.e.,

$X=\operatorname{Ker} M \oplus X_{2}, P, Q$ are two projectors satisfying $\operatorname{ImP}=\operatorname{KerM}, \quad \operatorname{Im} Q=Z_{1}, \quad N=N_{1}$, $\Sigma_{\lambda}=\left\{x \in \bar{\Omega}: M x=N_{\lambda} x\right\}$.

Lemma 2.1. ([15]) Let $X$ and $Z$ be two Banach spaces with norms $\|\cdot\|_{X},\|\cdot\|_{Z}$ respectively and $\Omega \subset X$ be an open and bounded nonempty set. Suppose $M: X \cap \operatorname{dom} M \rightarrow Z$ is quasi-linear and $N_{\lambda}: \bar{\Omega} \rightarrow Z, \quad \lambda \in[0,1]$ is $M-$ compact in $\bar{\Omega}$. In addition, if the following conditions hold:

$\left(\begin{array}{c}H_{1}\end{array}\right) \quad M x \neq N_{\lambda} x, \forall(x, \lambda) \in \partial \Omega \times(0,1) \quad ; \quad\left(H_{2}\right)$ $Q N x \neq 0, \forall x \in \operatorname{KerM} \cap \partial \Omega \quad ; \quad\left(\quad H_{3}\right)$ $\operatorname{deg}\{J Q N, \Omega \cap \operatorname{KerM}, 0\} \neq 0$,

$J: \operatorname{ImQ} \rightarrow \operatorname{KerM}$ is a homeomorphism. Then the abstract equation $M x=N x$ has at least one solution in $\operatorname{dom} M \cap \bar{\Omega}$.

\section{MAIN RESULTS}

For convenience of applying Lemma 2.3, we denote

$$
\begin{gathered}
X=C_{T}^{1}=\left\{x \mid x \in C_{r d}^{1}(T, R),\right. \\
\left.x(t+T)=x(t), x^{\Delta}(t+T)=x^{\Delta}(t)\right\}, \\
Z=C_{T}=\left\{x \mid x \in C_{r d}(T, R), x(t+T)=x(t)\right\}
\end{gathered}
$$

$\|x\|=\max \left\{\|x\|_{0},\left\|x^{\Delta}(t)\right\|_{0}\right\},\|x\|_{0}=\max _{t \in[0, T]}|x(t)|,[0, T]:=[0, T]_{R} \cap T$,

where $[0, T]_{R}$ denote the interval $[0, T]$ on $R$. Then the operators $M, N_{\lambda}$ are defined by

$$
\begin{gathered}
M: \operatorname{dom} M \cap X \rightarrow Z,(M x)(t)=\left(\varphi_{p}\left(x^{\Delta}(t)\right)\right)^{\Delta}, \\
N_{\lambda}: X \rightarrow Z,\left(N_{\lambda} x\right)(t)=-\lambda f(x(t)) X^{\Delta}(t)-\lambda g(x(t-\tau(t)))+\lambda e(t), \lambda \in[0,1]
\end{gathered}
$$

$$
\text { where } \quad \operatorname{dom} M=\left\{x \in X \mid \varphi_{p}\left(x^{\Delta}(t)\right) \in C_{T}^{1}\right\} \text {; }
$$$$
f, g \in C(R, R) \text {; }
$$$$
e, \tau \in C(T, R), e(t+T)=e(t), \tau(t+T)=\tau(t) \text {. Let }
$$$$
F\left(t, x(t), x^{A}(t), x(t-\tau(t))\right)=-f(x(t)) x^{A}(t)-g(x(t-\tau(t)))+e(t),
$$

then $N_{\lambda} X=\lambda F$. By (2) and (3), Eq. (1) is equivalent to the operator equation $M x=N x$, where $N_{1}=N$. Then we have

$$
\begin{gathered}
\operatorname{Ker} M=\{x \in X \mid x=a \in R\} \cong R, \\
\operatorname{Im} M=\left\{z \in Z \mid \int_{0}^{T} z(s) \Delta s=0\right\} .
\end{gathered}
$$

Then we have the following Lemma.

Lemma 3.1. Let $M$ be as defined by (2). Then $M$ is a quasi-linear operator. For all $t \in T$, define the operator $P, Q$ by

$P: X \rightarrow \operatorname{KerM},(P x)(t)=x(0), Q: Z \rightarrow R,(Q Z)(t)=\frac{1}{T} \int_{0}^{T} z(s) \Delta s$

Lemma 3.2. If $f, g \in C(R, R)$, and $e, \tau \in C(T, R)$ with $e(t+T)=e(t), \quad \tau(t+T)=\tau(t)$, then $N_{\lambda}$ is $M$ - compact.

Proof Let $Z_{1}=\operatorname{ImQ}$. For any bounded set $\bar{\Omega} \subset X \neq \varnothing, \quad$ define $\quad R: \bar{\Omega} \times[0,1] \rightarrow \operatorname{Ker} P$, $R(x, \lambda)(t)=\int_{0}^{t} \varphi_{p}\left[a_{x}+\int_{0}^{s} \lambda(F-Q F)(r) \Delta r\right] \Delta s, \lambda \in[0,1]$, where $F$ is defined by (3) $a_{x}$ is a constant related to $x$. Let

$G: R \rightarrow R, G(a)=\int_{0}^{t} \varphi_{p}\left[a+\int_{0}^{s} \lambda(F-Q F)(r) \Delta r\right] \Delta s$.

Since $G$ is continuous and increasing about $a$, and let

$$
\begin{aligned}
& A=\max _{s \in[0, T]} \int_{0}^{s} \lambda(F-Q F)(r) \Delta r, \\
& B=\min _{s \in[0, T]} \int_{0}^{s} \lambda(F-Q F)(r) \Delta r,
\end{aligned}
$$

then $G(-A) \leq 0, G(-B) \geq 0$, so we can choose $a$ satisfied $\quad G(a)=0$. From above, we know $a_{x} \in[-A,-B]$ exist and unique, so $R(x, \lambda)(t)$ is well defined. It is easy to prove that $R(x, \lambda)$ is relatively compact on $\bar{\Omega} \times[0,1]$.

Step 1. Since $Q^{2}=Q$, we have $Q(I-Q) N_{\lambda}(\bar{\Omega})=0$, so

$$
(I-Q) N_{\lambda}(\bar{\Omega}) \subset \operatorname{Ker} Q=\operatorname{Im} M .
$$

On the other hand, $\forall z \in \operatorname{Im} M$, clearly, $Q z=0$, so $z=z-Q z=(I-Q) z$, then $z \in(I-Q) Z$. So we have $(I-Q) N_{\lambda}(\bar{\Omega}) \subset \operatorname{ImM} \subset(I-Q) Z$.

Step 2. We show that: $Q N_{\lambda} x=0, \lambda \in(0,1) \Leftrightarrow Q N x=0, \forall x \in \Omega$. 
Step 3. When $\lambda=0$, since $a_{x} \in[-A,-B]$, then there exist $a_{x}=0$. For $a_{x}=0$, we have $R(x, 0)(t) \equiv 0$. $\forall x \in \Sigma_{\lambda}=\left\{x \in \bar{\Omega}: M x=N_{\lambda} x\right\}$, we have $\left(\varphi_{p}\left(x^{\Delta}(t)\right)\right)^{\Delta}=\lambda F$ and $Q F=\frac{1}{T} \int_{0}^{T}\left(\varphi_{p}\left(x^{\Delta}(t)\right)\right)^{\Delta} \Delta t=0$.

For $R(x, \lambda)(t)=\int_{0}^{t} \varphi_{q}\left[a_{x}+\int_{0}^{s} \lambda(F-Q F)(r) \Delta r\right] \Delta s$, take $a_{x}=-\varphi_{p}\left(x^{\Delta}(0)\right)$, we obtain

$$
\begin{gathered}
R(x, \lambda)(t)=\int_{0}^{t} \varphi_{q}\left[-\varphi_{p}\left(x^{\Delta}(0)\right)+\int_{0}^{s} \lambda(F-Q F)(r) \Delta r\right] \Delta s \\
=\int_{0}^{t} \varphi_{q}\left[\varphi_{p}\left(x^{\Delta}(s)\right)\right] \Delta s=x(t)-x(0)=(I-P) x(t) .
\end{gathered}
$$

Step 4. $\forall x \in \bar{\Omega}$, we have

$$
\begin{gathered}
M[P x+R(x, \lambda)](t) \\
=\left(\varphi_{p}\left(\left[x(0)+\int_{0}^{t} \varphi_{q}\left[-\varphi_{p}\left(x^{\Delta}(0)\right)+\int_{0}^{s} \lambda(F-Q F)(r) \Delta r\right] \Delta s\right)^{\Delta}\right)^{\Delta}\right. \\
=\left(\varphi_{p}\left(\varphi_{q}\left[-\varphi_{p}\left(x^{\Delta}(0)\right)+\int_{0}^{t} \lambda(F-Q F)(r) \Delta r\right]\right)^{\Delta}\right. \\
=\left[-\varphi_{p}\left(x^{\Delta}(0)\right)+\int_{0}^{t} \lambda(F-Q F)(r) \Delta r\right]^{\Delta} \\
=\lambda(F-Q F)(t)=\left(N_{\lambda}-Q N_{\lambda}\right) x(t) .
\end{gathered}
$$

Hence, $N_{\lambda}$ is $M-$ compact in $\bar{\Omega}$.

Theorem 3.1. Suppose $f, g \in C(R, R)$; $e, \tau(t) \in C(T, R)$ with

$e(t)=e(t+T)$ and $\tau(t)=\tau(t+T)$, there exist constant $d_{1}$, assume that the following conditions

(i) $f(u(t)) u^{\Delta}(t)>0$, when $|u(t-\tau(t))| \geq d_{1}$,

(ii) $\lim _{|u| \rightarrow+\infty} \frac{g(u)-|e|_{0}}{|u|}=r>0$,

(iii) $\lambda T^{\frac{p-2}{p-1}} \max |f(u)| \leq 1$, if $p=2$ hold. Then Eq. (1) has at least one T-periodic solution.

Proof We complete the proof by three steps.

Step

1.

Let $\Omega_{1}=\left\{x \in \operatorname{dom} M: M x=N_{\lambda} x, \lambda \in(0,1)\right\}$. We claim that $\Omega_{1}$ is a bounded set. If $x \in \Omega_{1}$, then $M x=N_{\lambda} x$, i.e.,

$$
\left(\varphi_{p}\left(x^{\Delta}(t)\right)\right)^{\Delta}+\lambda f(x(t)) x^{\Delta}(t)+\lambda g(x(t-\tau(t)))=\lambda e(t) .
$$

Integrating both sides of (5) over $[0, T]$, we have

$\int_{0}^{T} f(x(s)) x^{\Delta}(s) \Delta s=-\int_{0}^{T} g(x(s-\tau(s))) \Delta s+\int_{0}^{T} e(s) \Delta s$, that is,

$$
\int_{0}^{T} f(x(s)) x^{\Delta}(s) \Delta s=-\int_{0}^{T}[g(x(s-\tau(s)))-e(s)] \Delta s,
$$

then we have $\int_{0}^{T}\left[f(x(s)) x^{\Delta}(s)+g(x(s-\tau(s)))-e(s)\right] \Delta s=0$.

There must exist some $\xi$ such that

$$
f(x(\xi)) x^{\Delta}(\xi)+g(x(\xi-\tau(\xi)))-e(\xi) \leq 0 .
$$

From the assumption (i) and (ii), we have

$$
f(x(\xi)) x^{\Delta}(\xi)>0,|x(\xi-\tau(\xi))| \geq d_{1},
$$

and there exist constants $d_{2}>0$ and $\varepsilon>0$ such that $g(x(\xi-\tau(\xi)))-e(\xi)=(r+\varepsilon)|x(\xi-\tau(\xi))|>0,|x(\xi-\tau(\xi))| \geq d_{2}$.

So when $|x(\xi-\tau(\xi))| \geq d_{2}$, we obtain

$$
f(x(\xi)) x^{\Delta}(\xi)
$$

$\leq-g(x(\xi-\tau(\xi)))+e(\xi) \leq-g(x(\xi-\tau(\xi)))+|e|_{0} \leq 0$

Then we get $|x(\xi-\tau(\xi))| \leq \max \left\{d_{1}, d_{2}\right\}$, and

$|x(t)| \leq|x(\xi-\tau(\xi))|+\int_{0}^{T}\left|x^{\Delta}(s)\right| \Delta s \leq \max \left\{d_{1}, d_{2}\right\}+\int_{0}^{T}\left|x^{\Delta}(s)\right| \Delta s$.

Take the absolute value of both sides of the equation (3.4), and integrating it over $[0, T]$,

$$
\begin{aligned}
& \int_{0}^{T}\left|x^{\Delta}(s)\right|^{p-1} \Delta s \\
& \leq \lambda \int_{0}^{T}\left|f(x(s)) x^{\Delta}(s)\right| \Delta s+\lambda \int_{0}^{T}|[g(x(s-\tau(s)))-e(s)]| \Delta s \\
& \quad \leq \lambda \max _{t \in[0, T]}|f(x(t))| \int_{0}^{T}\left|x^{\Delta}(s)\right| \Delta s+\lambda T \max _{t \in[0, T]}|g(x(t-\tau(t)))-e(t)|
\end{aligned}
$$

By Hö lder inequality, and combining (7) we have

$$
\int_{0}^{T}\left|x^{\Delta}(s)\right| \Delta s \leq\left(\int_{0}^{T}\left|x^{\Delta}(s)\right|^{p-1} \Delta s\right)^{\frac{1}{p-1}} T^{\frac{p-2}{p-1}} .
$$

Substituting above inequality into (8) we obtain

$$
\begin{gathered}
\int_{0}^{T}\left|x^{\Delta}(s)\right|^{p-1} \Delta s \leq \lambda T^{\frac{p-2}{p-1}} \max _{t \in[0, T]}|f(x(t))|\left(\int_{0}^{T}\left|x^{\Delta}(s)\right|^{p-1} \Delta s\right)^{\frac{1}{p-1}} \\
+\lambda T \max _{t \in[0, T]}|g(x(t-\tau(t)))-e(t)| .
\end{gathered}
$$

Since $\frac{1}{p-1} \leq 1$, so $\left|x^{\Delta}(t)\right|$ bounded, that means there exist $M_{2}$ such that $\left|x^{\Delta}(t)\right| \leq M_{2}$, so we have $|x(t)| \leq \max \left\{d_{1}, d_{2}\right\}+T M_{2}:=M_{0}$.

Step 2 Let $\Omega_{2}=\{x \in \operatorname{KerM}: Q N x=0\}$. For $\forall x \in \Omega_{2}$, then

$$
x(t)=a_{0} \in R
$$

Since

$N x=-f(x(t)) x^{\Delta}(t)-g(x(t-\tau(t))+e(t)$, we have $Q N x=\frac{1}{T} \int_{0}^{T} N x(s) \Delta s=\frac{1}{T} \int_{0}^{T}\left[-g\left(a_{0}\right)+e(s)\right] \Delta s=-g\left(a_{0}\right)+\frac{1}{T} \int_{0}^{T} e(s) \Delta s=0$.

From the assumption, $\lim _{|u| \rightarrow+\infty} \frac{g(u)-|e|_{0}}{|u|}=r>0$, we have $\left|a_{0}\right| \leq d_{2}$. Take the open and bounded set 
$\Omega \supset \Omega_{1} \cup \Omega_{2}$, then the conditions $\left(H_{1}\right)$ and $\left(H_{2}\right)$ of Lemma 2.3 satisfied.

$$
\begin{array}{ccc}
\text { Step } & 3 & \text { Define } \\
J: \operatorname{ImQ} & \rightarrow \operatorname{KerM}, J(a)=a, a \in R & \text { Take }
\end{array}
$$

operator

$$
H(x, \mu)=\mu a_{0}-(1-\mu) J Q N x,
$$

then

$$
\begin{gathered}
H\left(a_{0}, \mu\right)=a_{0} \mu-(1-\mu)\left(-g\left(a_{0}\right)+\int_{0}^{T} e(s) \Delta s\right), \\
a_{0} H(x, \mu)=\mu a_{0}^{2}-(1-\mu) a_{0}\left(-g\left(a_{0}\right)+\int_{0}^{T} e(s) \Delta s\right)>0 .
\end{gathered}
$$

So

$H\left(a_{0}, \mu\right) \neq 0$.

$\operatorname{deg}\{J Q N, \Omega \cap \operatorname{KerM}, 0\}=\operatorname{deg}\{-I, \Omega \cap \operatorname{KerM}, 0\} \neq 0$.

From the above prove, we can get the fact that Eq. (1) has at least one $T$ - periodic solution. The proof is completed.

Theorem 2. Assume the condition (ii) of Theorem 3.1 holds, and the following conditions satisfied:

(iv) there exists a continuous function $c(t)$ on time scales $T$ satisfies

$$
-f(u(t)) u^{\Delta}(t)-g(u(t-\tau(t))+e(t) \geq c(t) .
$$

(v) there exists a constant $R_{1}>0$ such that

$$
\int_{0}^{T}\left[-f(u(t)) u^{\Delta}(t)-g(u(t-\tau(t))+e(t)] \Delta t>0, u_{L} \geq R_{1},\left|u^{\Delta}(t)\right| \leq M,\right.
$$

and

$$
\int_{0}^{T}\left[-f(u(t)) u^{\Delta}(t)-g(u(t-\tau(t))+e(t)] \Delta t<0, u_{M} \leq-R_{1},\left|u^{\Delta}(t)\right| \leq M .\right.
$$

Then Eq. (1) has at least one $T$ - periodic solution. Where

$$
u_{L}:=\min _{t \in[0, T]} u(t), u_{M}:=\max _{t \in[0, T]} u(t) .
$$

Proof

Step

1.

Let

$\Omega_{1}=\left\{x \in \operatorname{dom} M: M x=N_{\lambda} x, \lambda \in(0,1)\right\}$. We show that $\Omega_{1}$ is a bounded set. If $x \in \Omega_{1}$, then $M x=N_{\lambda} x$, i.e.,

$$
\left(\varphi_{p}\left(x^{\Delta}(t)\right)\right)^{\Delta}+\lambda f(x(t)) x^{\Delta}(t)+\lambda g(x(t-\tau(t)))=\lambda e(t) .
$$

From the definition of operator $Q$, we know that $Q M x(t)=Q N_{\lambda} x(t)=0$, that is $Q N x(t)=0$. The operator $N x$ is bounded from below by $c$ on $T$, so we have the inequality: $|N x(t)| \leq N x(t)+2 c^{-}(t), \forall t \in[0, T]$, where we denote $c^{-}(t)=\max \{-c(t), 0\}$. Combining the condition (iv) we obtain
$\int_{0}^{T}\left|\left(\varphi_{p}\left(x^{\Delta}(t)\right)\right)^{\Delta}\right| \Delta t=\lambda \int_{0}^{T}|N x(t)| \Delta t \leq \int_{0}^{T} N x(t) \Delta t+2 T\left|c^{-}(t)\right|_{0}=2 T\left|c^{-}\right|_{0}$,

that is $\int_{0}^{T}\left|x^{\Delta}(t)\right|^{p-1} \Delta t \leq 2 T\left|c^{-}\right|_{0}$, then there exist a constant $M_{2}$ such that $\left|x^{\Delta}(t)\right| \leq M_{2}$. From the condition (v), if $x_{L} \leq R_{1}$ ( or $x_{M} \geq R_{1}$ ), we know

$$
\begin{gathered}
\int_{0}^{T} N x(t) \Delta t<0\left(\text { or } \int_{0}^{T} N x(t) \Delta t>0\right), \text { so } \\
x_{M}>-R_{1}\left(\text { or } x_{L}<R_{1}\right) .
\end{gathered}
$$

Clearly, we have $x_{M} \leq x_{L}+\int_{0}^{T}\left|x^{\Delta}(t)\right| \Delta t$. We can get

$$
-\left(R_{1}+M_{2} T\right)<x_{L}<x_{M}<R_{1}+M_{2} T,
$$

that means $|x|_{0}<R_{1}+M_{2} T$. The next two steps are similar to the proof of Theorem 3.1, and then we can obtained Eq. (1) has at least one $T$ - periodic solution. The proof is completed.

\section{ACKNOWLEDGEMENTS}

This research is supported by the NSF of China (61374074), and supported by NSF of Shandong Provincial (ZR2011AL007, ZR2012AM009, ZR2013AL003).

*Corresponding author: Zhenlai Han, e-mail: hanzhenlai@163.com.

\section{REFERENCES}

[1] Bing Xiao, Bingwen Liu. Periodic solutions for Rayleigh type $p$ Laplacian equation with a deviating argument. Nonlinear Analysis: Real World Applications, 10, pp. 16-22, 2009.

[2] Cristian Bereanu, Jean Mawhin. Periodic solutions of nonlinear perturbations of $\phi-$ Laplacians with possibly bounded $\phi$. Nonlinear Analysis, 68, pp. 1668-1681, 2008.

[3] Wing-Sum Cheung, Jingli Ren. On the existence of periodic solutions for $p$-Laplacian generalized Li é nard equation. Nonlinear Analysis, 60, pp. 65-75, 2005.

[4] Fengjuan Cao, Zhenlai Han, Shurong Sun. Existence of periodic solutions for a fourth-order $p$-Laplacian differential equation. Acta Scientiarum Naturalium Universitatis Sunyatseni, 49 (3), pp. 18-23, 2010.

[5] Fengjuan Cao, Zhenlai Han, Ping Zhao, Shurong Sun. Uniqueness of periodic solution for a class of Li é nard $p$-Laplacian equations. Advances in Difference Equations, 2010, Article ID 235749, pp. 114, 2010.

[6] R. Man á sevich, J. Mawhin. Periodic solutions for nonlinear systems with $p$-Laplacian-like operators. J. Differential Equations, 145 , pp. 367-393, 1998

[7] Wing-Sum Cheung, Jingli Ren. Periodic solutions for $p$-Laplacian Li e nard equation with a deviating argument. Nonlinear Analysis, 59, pp. 107-120, 2004.

[8] Shiping Lu. Existence of periodic solutions to a p-Laplacian Li é nard differential equation with a deviating argument. Nonlinear Analysis, 68, pp. 1453-1461, 2008.

[9] Bo Du, Xiangkui Zhao. A new method for the existence of periodic solution to a $p$-Laplacian Li e nard equation. J. Appl. Math. Comput., 29, pp. 481-490, 2009. 
[10] E. R. Kaufmann, Y. N. Raffoul. Periodic solutions for a neutral nonlinear dynamical equation on a time scales. J. Math. Anal. Appl., 319, pp. 315-325, 2006.

[11] Yongkun Li, Hongtao Zhang. Existence of periodic solutions for a periodic mutualism model on time scales. J. Math. Anal. Appl, 343, pp. 818-825, 2008.

[12] Martin Bohner, Meng Fan, Jimin Zhang. Existence of periodic solutions in predator-prey and competition dynamic systems. Nonlinear Analysis: Real World Applications, 7, pp. 1193-1204, 2006.
[13] M. Bohner. A.:Peterson, Advances in Dynamic Equations on Time Scales, Birkh $\ddot{a}$ user, Boston, 2003.

[14] Fengjuan Cao, Zhenlai Han, Shurong Sun. Existence of periodic solutions for p-Laplacian equations on time scales. Advances in Difference Equations, 2010, Article ID 584375, pp. 1-13,2010.

[15] Weigao Ge, Jingli Ren. An extension of Mawhin's continuation theorem and its application to boundary value problems with a $p$ Laplacian. Nonlinear Analysis, 58, pp. 477-488, 2004. 